\title{
Seeing the body modulates audiotactile integration
}

\author{
Jane E. Aspell, ${ }^{1}$ Tom Lavanchy, ${ }^{1}$ Bigna Lenggenhager ${ }^{1}$ and Olaf Blanke ${ }^{1,2}$ \\ ${ }^{1}$ Laboratory of Cognitive Neuroscience, Ecole Polytechnique Fédérale de Lausanne (EPFL), Swiss Federal Institute of Technology, \\ 1015 Lausanne, Switzerland \\ ${ }^{2}$ Department of Neurology, University Hospital, Geneva, Switzerland
}

Keywords: audiotactile, body, crossmodal congruency effect, multisensory, vision

\begin{abstract}
Audiotactile integration has been studied using various experimental setups but so far crossmodal congruency effects (CCEs) have not been found for tactile targets paired with auditory distractors. In the present study we investigated whether audiotactile CCEs exist and, if so, whether these CCEs have similar characteristics to those found by previous authors with visual distractors. We measured audiotactile CCEs by attaching four vibrators to the backs of participants and presented auditory stimuli from four loudspeakers placed, in separate blocks, at different distances in front of or behind the participant's body. Participants discriminated the elevation of tactile stimuli while ignoring the auditory distractors. CCEs were found only when participants were provided with noninformative vision of their own body, as seen from behind via a camera and head-mounted display; they were absent when participants did not view their body. Furthermore, in contrast to visuotactile CCEs, audiotactile CCEs did not depend on whether the distractors were presented on the same or different side as the tactile targets. The present study provides the first demonstration of an audiotactile CCE: incongruent auditory distractors impaired performance on a tactile elevation discrimination task relative to performance with congruent distractors. We show that audiotactile CCEs differ from visuotactile CCEs as they do not appear to be as sensitive to the spatial relations between the distractors and the tactile stimuli. We also show that these CCEs are modulated by vision of the body.
\end{abstract}

\section{Introduction}

The ubiquity of multisensory processing in the brain is demonstrated by the ever-increasing number of studies on this topic (for reviews see, e.g., Welch \& Warren, 1986; Stein \& Meredith, 1993; Driver \& Spence, 1998a; Schroeder \& Foxe, 2005). Research on multisensory processing has mainly focused on audiovisual and visuotactile interactions; audiotactile processing has received less attention. Audiotactile integration differs from other types of multisensory interactions in some respects, and some of the principles of audiotactile integration have recently been described (Jousmäki \& Hari, 1998; Foxe et al., 2000; Caclin et al., 2002; Farne \& Ladavas, 2002; Lütkenhöner et al., 2002; Bresciani et al., 2005; Kayser et al., 2005; Kitagawa \& Spence, 2006; Schürmann et al., 2006; Zampini et al., 2007; Soto-Faraco \& Deco, 2009; Tajadura-Jiménez et al., 2009). These studies have shown that, whereas vision is dominant in spatial tasks involving audiovisual stimuli, there is no obvious pattern of modality dominance in audiotactile spatial tasks (e.g. Caclin et al., 2002). Furthermore, although audiovisual interactions are influenced by spatial relations (Welch \& Warren, 1986; Stein \& Meredith, 1993), they appear to be less dependent on spatial coincidence (but more so on temporal stimulus attributes: Jousmäki \& Hari, 1998; Lloyd et al.,

Correspondence: Dr Jane E. Aspell, Laboratory of Cognitive Neuroscience, Brain Mind Institute, SV 2805, Station 19, as above.

E-mail: jane.aspell@epfl.ch

Received 2 October 2009, revised and accepted 11 February 2010
2003; Zampini et al., 2005). The influence of spatial relations on audiotactile interactions seems to depend on the region of space that is stimulated: there is evidence that the rear space is the region in which audiotactile spatial interactions are most prevalent, probably because vision is of no use here (Graziano et al., 1999; Farne \& Ladavas, 2002; Fu et al., 2003; Kitagawa et al., 2005; Zampini et al., 2005, 2007; Kitagawa \& Spence, 2006).

One well-studied multisensory paradigm for investigating how spatial relations affect visuotactile integration is the crossmodal congruency task (Spence et al., 2004). Most previous studies of the crossmodal congruency effect (CCE) have examined interactions between stimuli on or near the hands (Pavani et al., 2000; Austen et al., 2004; Pavani \& Castiello, 2004; Spence et al., 2004; Shore et al., 2006; Igarashi et al., 2008). Participants perform worse when a distracting visual stimulus occurs at an incongruent elevation with respect to the tactile (target) stimulus. Interestingly, the CCE (the difference between performance in incongruent and congruent conditions) is larger when the visual and tactile stimuli occur closer to each other in space (Spence et al., 2004). To date, there have been no complete published studies demonstrating a crossmodal congruency effect for audiotactile stimuli. Lloyd et al. (2003) used a variant of the crossmodal congruency task to investigate crossmodal spatial attention effects between audition and touch, but they did not investigate nor report congruency effects per se. In the Lloyd et al. (2003) study, targets could be auditory or tactile and participants were instructed to respond to their elevation, regardless of modality. The data showed 
that participants could spatially shift their attention in one modality independently of the other and could also direct both tactile and auditory attention to a common side of space.

Here we extended a recently developed visuotactile crossmodal congruency task for the full body (Aspell et al., 2009) to the study of audiotactile spatial interactions. Our goal was to investigate whether CCEs could be found for tactile targets paired with auditory distractors and if so, whether these audiotactile CCEs would have similar characteristics to those found with visual distactors. In addition, we aimed to test the effect of the location (front vs. rear space) of auditory distractors on CCEs, predicting that they would be larger for rear space auditory stimuli. A number of studies have found that audiotactile integration is more pronounced when auditory stimuli are closer to the body (Graziano et al., 1999; Kitagawa et al., 2005; Menning et al., 2005) and others found that audiotactile interactions are more pronounced with rear-space auditory stimuli (Farne \& Ladavas, 2002; Fu et al., 2003). We also tested whether noninformative vision of the body would have any effect on the magnitude of audiotactile CCEs, predicting that it would increase their magnitude, given recent results on visuotactile CCEs (Aspell et al., 2009).

\section{Materials and methods \\ Participants}

A total of 37 participants took part in three experiments: 14 (seven females; mean age $26 \pm 2$ years) in Experiment 1; 10 (three females; mean age $25 \pm 3$ years) in Experiment 2 and 13 (five females; mean age $24 \pm 3$ years) in Experiment 3. All participants had normal vision and hearing and had no history of neurological or psychiatric conditions. They gave written informed consent and were renunerated for their participation. The study protocol was approved by the local ethics research committee at the University of Lausanne (Switzerland) and the study conforms to The Code of Ethics of the World Medical Association (Declaration of Helsinki) printed in the British Medical Journal (18 July 1964).

\section{Materials}

The experiments were run in an experimental room under artificial light. Stimulus presentation and timings were controlled using the E-PRIME software (Psychology Software Tools Inc., Pittsburgh, PA, USA). Some of the material was as described previously (Aspell et al., 2009) and the set-up was adapted for the present study as described below. Previous (visuotactile) CCEs used relatively short stimulus durations (typically $30-50 \mathrm{~ms}$ ) and stimulus onset asynchronies of 30-100 ms (Spence et al., 2004; Shore et al., 2006). These parameters were shown to elicit maximal CCEs and were used as an initial guide. In the present experiments reported here we used a somewhat longer duration for the vibration stimulus as the vibrations were delivered to the back (rather than to the fingers as in most previous studies), which is less sensitive to tactile stimulation. Optimal timings and stimulus locations were determined in pilot tests in which we checked whether participants were able to discriminate the elevation of the vibrations and of the sounds by measuring accuracy when presenting each stimulus type separately. We attached four vibration devices, each consisting of a small vibrating motor, to the skin of participants' backs using tape. The two 'upper' devices were positioned at the inner edges of the shoulder blades ( $20 \mathrm{~cm}$ apart) and the two 'lower' devices $9 \mathrm{~cm}$ below. For auditory stimulus presentation we used four loudspeakers (model MLS 3 2W, 8 OHM, 66 mm diameter, Velleman, Gavere, Belgium) emitting white noise bursts at $120 \mathrm{~dB}$. In all experiments, the loudspeakers were placed at lateral distances of $60 \mathrm{~cm}$ from the participants' bodies. The speakers were located on wooden supports at the same approximate elevation (height) as the four vibrators on the body. The speakers could be placed at different heights on the supports (depending on the height of each participant) and these supports could be placed at different distances in front or behind the participant (see Fig. 1).

As we did not find any significant audiotactile congruency effects in a pilot study using the same experimental setup but in which participants $(n=11)$ viewed a wall during the experiment, we adapted the pilot experimental set-up so that participants could view their own bodies (note that this visual information was noninformative concerning the audiotactile task). We aimed to test whether audiotactile CCEs could be found when participants viewed their own body during the task. A previous study from our lab (Aspell et al., 2009) showed that visuotactile CCEs were larger when participants were provided with noninformative vision of their bodies (their backs and heads) during the task; we thus predicted that viewing one's body might also facilitate audiotactile interactions, leading to an audiotactile CCE. Participants were therefore fitted with a head-mounted display (HMD) linked to a camera that filmed their bodies in real time from $2 \mathrm{~m}$ behind (see Fig. 1). In order to investigate whether the audiotactile CCEs were dependent on the vision of a human body (as shown by visuotactile studies: Tipper et al., 1998; Kennett et al., 2001; Aspell et al., 2009) we ran a control experiment (Experiment 2) in which participants could see the loudspeakers and the background via the HMD but not their body.

Given the results of the first two experiments (see Results section below: the finding of an audiotactile CCE only in the first experiment when the body was visible) we performed Experiment 3 in which we combined the conditions from Experiments 1 and 2 in a single experiment with a single group of participants, rendering analysis and interpretation clearer.

In the current study we tested audiotactile CCEs using three different sound locations: we placed the loudspeakers at $50 \mathrm{~cm}$ behind or at $50 \mathrm{~cm}$ or $150 \mathrm{~cm}$ in front of the participants. The 'front $50^{\prime}$ condition was the opposite of the 'back 50' condition. The 'front 150 ' distance was included because it has been previously shown that participants may perceive themselves to be at the location where they view their body, i.e. if they see their body in front of them they feel as if they are located in that position (Aspell et al., 2009). In the body-visible conditions the body was viewed $200 \mathrm{~cm}$ in front. If participants perceived themselves at that position in the present experiments as well, the ' $150 \mathrm{~cm}$ in front position' would be equivalent to $50 \mathrm{~cm}$ behind the perceived position (as a '200 cm in front position' would be equivalent to the seen position).

Each trial consisted of a single white noise burst followed by a vibrotactile stimulus. The active loudspeaker (i.e. the one that emitted the sound) and the active vibrating motor (the one that vibrated) were varied randomly and independently from trial to trial. Each trial began with a white-noise burst of $40 \mathrm{~ms}$ duration and was followed, with a stimulus onset asynchrony of $56 \mathrm{~ms}$ duration, by a vibrotactile stimulus of $70 \mathrm{~ms}$ duration. We presented the auditory stimulus first because in previous crossmodal congruency studies the distractor was almost always presented first, as this has been shown to elicit the maximal CCEs (Spence et al., 2004; Shore et al., 2006). Each experimental block contained 80 trials.

\section{Procedure}

The procedure was identical for all experiments except for those details added below. For each trial, participants had to signal with 

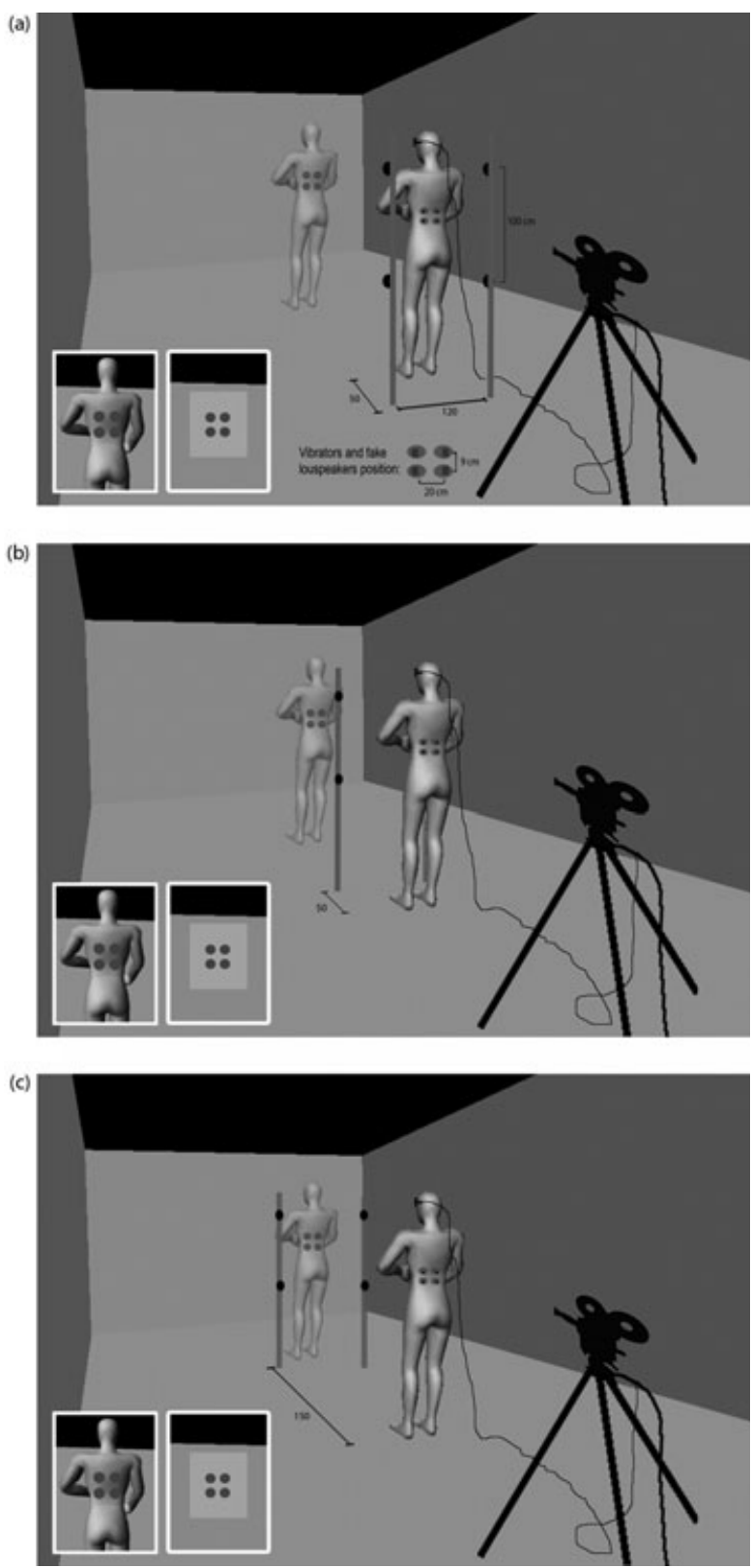

FIG. 1. Experimental set-up. Participants were standing and four loudspeakers were either located (a) $50 \mathrm{~cm}$ behind, (b) $50 \mathrm{~cm}$ in front or (c) $150 \mathrm{~cm}$ in front of their bodies. Four vibration devices were fixed to the participant's back, the upper two at the inner edges of the shoulder blades and the lower two $9 \mathrm{~cm}$ below. Four small fake loudspeakers were also attached to participants' backs and a video camera filmed the participants from behind. The 'real' loudspeakers were never visible. The small inset windows represent what the participants viewed via the camera and the HMD: either their own body with fake loudspeakers attached (left) or just the fake loudspeakers on a textured background (right).

their right hand, by pressing one of two buttons on a response box as fast as possible, whether they felt a vibration at the top (an upper device) or at the bottom (a lower device) of their backs (regardless of side), while trying to ignore the white noise bursts. There was a $1000-\mathrm{ms}$ pause between the participant's response and the succeeding trial. The setup and the instructions were thus similar to those employed in previous visuotactile CCE studies (Pavani et al., 2000; Spence et al., 2004; Aspell et al., 2009).
Reaction times and accuracies were recorded. The different blocks were presented in a random order. The speakers were repositioned between blocks when necessary.

\section{Experiment 1}

Experiment 1 investigated whether audiotactile CCEs would be found when viewing one's own body during the audiotactile task. Four small fake loudspeakers were attached to their backs in order to give participants the impression that the auditory stimuli were close in space to the vibrotactile stimuli. Within this set-up, the participants could not see the 'real' active loudspeakers; accordingly, there were no differing visual cues for the conditions in which the active loudspeakers were at different distances. Participants had a restricted field of view and could only see their bodies and the attached fake loudspeakers (see Fig. 1). Participants stood during the experimental blocks and fixated a point in the middle of their backs between the fake loudspeakers. The real (active) loudspeakers were positioned either in the rear space or in the front space. They were always vertically and laterally centred on the vibrators ("back level"). In the rear space, the speakers were placed at $50 \mathrm{~cm}$ distance (back 50 position) and, in the front space, at 50 or $150 \mathrm{~cm}$ distance (front 50 and front 150 positions).

\section{Experiment 2}

In this experiment, which was a control for experiment 1 , the body was not visible. The set-up was the same as that in experiment 1 except that the HMD projected a previously recorded image of the four fake loudspeakers, seen against a grey textured wall (i.e. presented in the absence of a body).

\section{Experiment 3}

In this experiment we tested two locations and two visual conditions (body visible or not visible). The setup was the same as before and the following four conditions were presented in a single session, in randomised order across participants: (i) body visible with front 50 loudspeakers, (ii) body visible with back 50 loudspeakers, (iii) body not visible with front 50 loudspeakers and (iv) body not visible with back 50 loudspeakers.

\section{Statistical analysis}

Trials with incorrect responses and trials in which participants failed to respond within $1500 \mathrm{~ms}$ were discarded from the analyses (following the methods of Spence et al., 2004; and Aspell et al., 2009). Inverse efficiency (IE) was calculated for each experimental condition and for each participant by dividing the mean reaction time by the percentage of correct responses in that condition. This has been shown to be a good index of behavioural performance as it combines response speed and accuracy and thus provides some correction for potential speed-accuracy tradeoffs in the data (Townsend \& Ashby, 1983; Spence et al., 2001). The mean IEs were found to have equal variances (Mauchly's test for sphericity) and were analyzed using two-tailed repeated-measures ANOVAs. For experiments 1 and 2 we ran repeated-measures ANOVAs on each of the three conditions (back 50, front 50 and front 150) with factors side (same, different) and congruency (congruent, incongruent). For experiments 1 and 2 the significance level was corrected for multiple comparisons $(P<0.017)$. For experiment 3 we ran a single repeatedmeasures ANOVA with factors congruency (congruent, incongruent), side (same, different), location (front 50, back 50) and body (visible, not visible). 


\section{Results}

\section{Experiment 1 (Body visible)}

Figure 2 plots the size of the CCE for the back 50, front 50 and front 150 conditions. Mean accuracy was similar in all conditions: $92 \%$ in the back 50 condition, $92 \%$ in the front 50 condition and $93 \%$ in the front 150 condition. Statistical analysis revealed for the front 50 condition a significant main effect of congruency $\left(F_{1,13}=8.34\right.$, $P=0.013)$ but no significant main effect of side $\left(F_{1,13}=1.04\right.$, $P=0.326)$. For the front 150 condition no significant main effect of congruency $\left(F_{1,13}=2.92, \quad P=0.111\right)$ or of side was found $\left(F_{1,13}=6.29, P=0.026\right)$. For the back 50 condition no significant main effect of congruency $\left(F_{1,13}=4.55, P=0.053\right)$ or of side was found $\left(F_{1,13}=4.32, P=0.058\right)$. No significant interactions were found for any conditions.

\section{Experiment 2 (Control experiment: no body visible)}

Figure 3 plots the size of the CCE for the back 50, front 50 and front 150 conditions. Mean accuracy was similar in all conditions ( $86 \%$ in the back 50 condition, $89 \%$ in the front 50 and the front 150 conditions). Statistical analysis revealed no significant congruency effects in any conditions $\left(F_{1,9}=2.13, P=0.178\right.$ for the back 50 condition, $F_{1,9}=0.39, P=0.544$ for the front 50 condition, $F_{1,9}=0.01, P=0.94$ for the front 150 condition). No significant side effects were found in the front 50 condition $\left(F_{1,9}=6.82, P=0.028\right)$, in the back $50\left(F_{1,9}=2.76, P=0.131\right)$ or in the front 150 condition $\left(F_{1,9}=1.77, P=0.216\right)$. No significant interactions were found in this experiment.

\section{Experiment 3 (Body visible or not visible)}

Figure 4 plots the size of the CCE for the back 50 and front 50 conditions when the body could be seen (Fig. 4a) or could not be seen (Fig. 4b). Mean accuracy was similar in all conditions $(95 \%$ for body visible, 50 back; $94 \%$ for body visible, 50 front; $96 \%$ for body not visible, 50 back; and $97 \%$ for the body not visible, 50 front condition). Statistical analysis revealed significant main effects of congruency $\left(F_{1,12}=6.74 ; P=0.023\right)$ and of side $\left(F_{1,12}=16.33\right.$; $P=0.002)$ and a significant interaction between body and congruency $\left(F_{1,12}=5.85 ; P=0.032\right)$. No other main effects or interactions reached significance $(P>0.05)$. Given the body $\times$ congruency interaction we ran post hoc (Tukey's HSD) tests and these revealed a significant difference between congruent and incongruent IEs in the

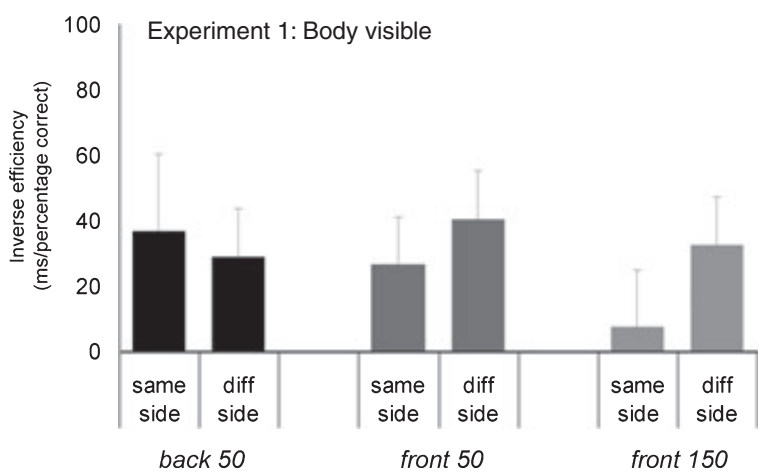

FIG. 2. Mean congruency effects (inverse efficiency in incongruent trials minus inverse efficiency in congruent trials) in experiment 1 for back 50 , front 50 and front 150 conditions. Error bars show SEM and units are $\mathrm{ms} /$ percentage correct.

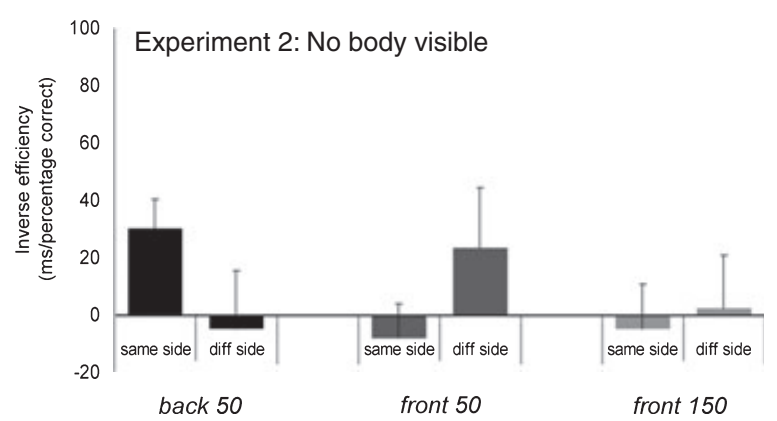

FIG. 3. Mean congruency effects (inverse efficiency in incongruent trials minus inverse efficiency in congruent trials) in experiment 2 for back 50 , front 50 and front 150 conditions. Error bars show SEM and units are $\mathrm{ms} /$ percentage correct.
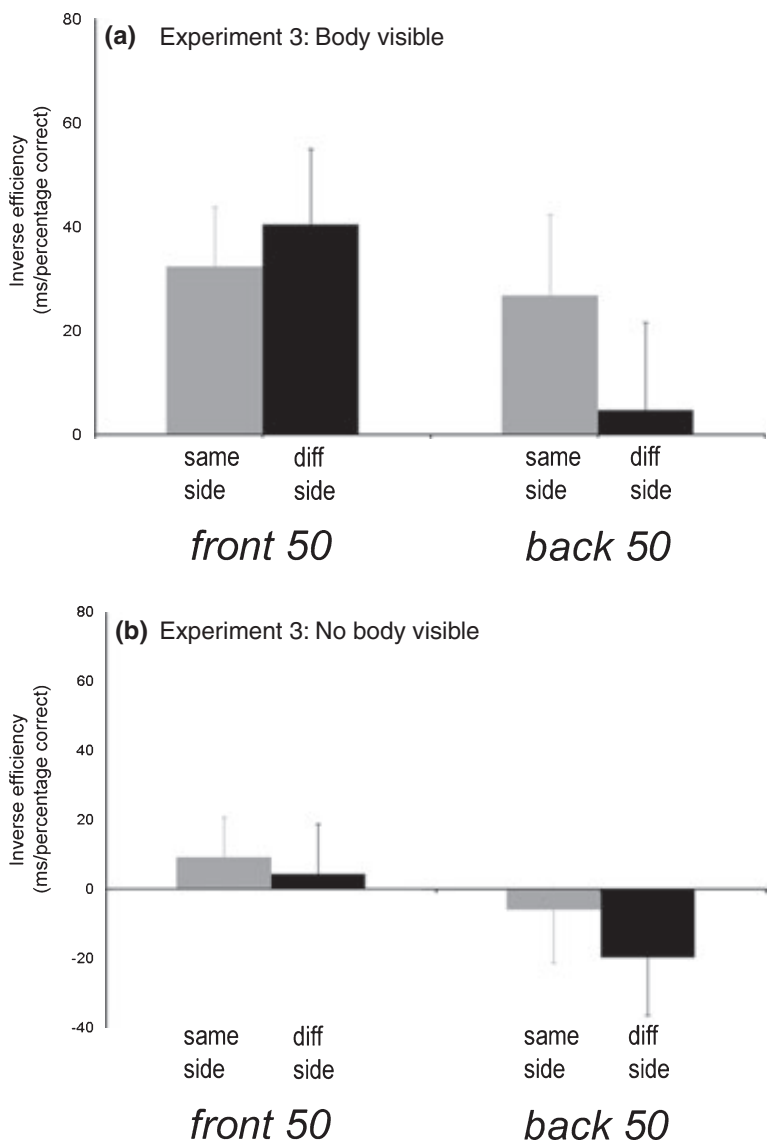

FIG. 4. Mean congruency effects (inverse efficiency in incongruent trials minus inverse efficiency in congruent trials) in experiment 3 for body visible back 50 and front 50 and conditions (a) and for body not visible back 50 and front 50 and conditions (b). Error bars show SEM and units are ms/percentage correct.

'body visible' condition $(P=0.003)$ but not in the 'body not visible' condition $(P=0.682)$

\section{Summary of results}

Audiotactile CCEs were found only in the front 50 condition of experiment 1 in which participants were able to view their own body. Experiment 2 was a control for experiment 1: the participant's body could not be seen via the HMD; under these conditions no audiotactile CCEs were found. Experiment 3 revealed that audiotactile CCEs were 
larger when the body was seen than when it was not seen (a significant interaction between congruency and body factors was found, and a significant congruency effect was only found for the body visible conditions). Note that in none of the experiments did we find an interaction between congruency and side as has been generally reported for visuotactile CCEs (Spence et al., 2004; Aspell et al., 2009).

\section{Discussion}

\section{Audiotactile CCES}

The present study demonstrates the existence of audiotactile crossmodal congruency effects: incongruent auditory distractors impaired performance on a tactile elevation discrimination task relative to performance with congruent distractors. This auditory effect on tactile perception was only found when participants were provided with noninformative vision of their own body as seen from behind (experiments 1 and 3) and was absent in experiment 2 when participants did not view their body (experiments 2 and 3).

In the present study the tactile stimuli were delivered to the back of participants but we note that CCEs have mostly been studied when visual and tactile stimuli were delivered to participants' hands. Nevertheless, recent studies have reported CCEs when (visual) distractors were not located on the body but at some distance from it, e.g. two studies (Pavani et al., 2000; Austen et al., 2004) found a CCE when the visual stimuli were presented on rubber hands. Visuotactile CCEs have also been observed for shadows of hands (Pavani \& Castiello, 2004), for photographs of hands presented via video monitors (Igarashi et al., 2008) and when the body was viewed from behind as in the present setup (Aspell et al., 2009). Moreover, visuotactile CCEs have also been found when the tactile stimuli were delivered to the back, which was seen on an HMD (Aspell et al., 2009). Thus it does not seem to be crucial that the targets are presented on the hands during crossmodal congruency tasks.

Regarding sound location, we did not find strong evidence for an effect of front vs. rear sounds on audiotactile CCEs. Although in experiment 1 CCEs were only found for the front 50 condition, the directly comparable data in experiment 3 failed to reveal any difference depending on whether sounds were presented in front or rear space. A significant side effect was found in experiment 3 , due to larger IEs for different side than for same side, as has been found in previous visuotactile CCE studies.

\section{Viewing the body}

In the present study audiotactile CCEs were observed only when the body was visible (experiment 1 and body visible conditions of experiment 3). The visual input was noninformative in terms of the varying target and distractor locations so it is perhaps surprising that it should affect audiotactile interactions. Nevertheless, it has previously been shown that viewing the body can affect tactile representation (Tipper et al., 1998, 2001; Whiteley et al., 2004, 2008; Haggard et al., 2007). The present data suggest that such effects of noninformative bodily vision extend to audiotactile representations. In a recent study (Aspell et al., 2009) we found that viewing one's own body from behind (in a very similar setup) led to greater visuotactile CCEs than when the body could not be seen.

The effect of seeing the body on audiotactile CCEs can be compared to the effects of seeing hands, hand-like objects and bodies on visuotactile CCEs (Pavani et al., 2000; Austen et al., 2004; Pavani \& Castiello, 2004; Igarashi et al., 2008; Aspell et al., 2009). The important difference in the present study is that auditory distractors are shown to influence the perception of tactile targets when specific information about the body from a third modality, vision, is added. To the best of our knowledge, the present study is the first demonstration in humans of a modulation of audiotactile interactions by vision of the body. This could be because the visual input provides a reference frame for audiotactile integration, or it may be due to visual capture of the tactile targets, and/or it may be that vision of the body activates a body representation (see Kennett et al., 2001; Whiteley et al., 2008) which then facilitates multisensory audiotactile interactions between stimuli attributed to the body and/or those stimuli in peripersonal or near-personal space.

In experiment 2 (a control for experiment 1) participants viewed the same fake loudspeakers as in experiment 1 but this time without also seeing their body. With this setup no audiotactile CCEs were found. This effect was replicated in an independent study sample (experiment 3 ) in which the body visible and not visible conditions were tested in the same session and tested on the same participants. It seems, therefore, that the presentation of a structured visual environment via an HMD is not sufficient to facilitate audiotactile integration because audiotactile CCEs were only found when the body was seen (see Warren, 1970).

Why should vision influence audiotactile integration or, more generally, why should noninformative signals from a third modality influence bimodal interactions? Previous studies have shown that bimodal integration can be influenced by signals from a third modality, e.g. visuotactile integration can be modulated by crossing the arms (demonstrating an influence of proprioception; see e.g. Driver \& Spence, 1998b). Moreover, 'trimodal' interactions are consistent with neurophysiological findings of the existence of trimodal neurons, e.g. neurons responding to tactile, auditory and visual stimuli can be found in several brain regions including ventral premotor cortex (Graziano et al., 1999), the ventral intraparietal and temporoparietal areas (Leinonen et al., 1980; Ghazanfar \& Schroeder, 2006), the superior temporal sulcus (Bruce et al., 1981) and the superior colliculus (Wallace et al., 1993).

\section{Comparison with visuotactile CCEs}

The audiotactile CCEs demonstrated here were of lower magnitude than those typically reported for visuotactile interactions. Visuotactile CCEs (reported as IE scores) have been found to be in the range 85$150 \mathrm{~ms} /$ percentage correct (Shore et al., 2006; Schicke et al., 2009) yet the corresponding values for the audiotactile CCEs were smaller, averaging maximally $\sim 40 \mathrm{~ms} /$ percentage correct. The smaller audiotactile CCE may be partly due to the poorer spatial resolution of audition than of vision (Welch \& Warren, 1986). It is likely that incongruent and congruent auditory distractors cannot be as well differentiated (Blauert, 1997) as incongruent and congruent visual distractors and therefore can only differentially affect tactile discrimination to a lesser degree. This is compatible with the finding in related behavioural paradigms that audiotactile interactions are generally weaker and less prevalent than visuotactile or audiovisual interactions (e.g. Lloyd et al., 2003).

Studies of visuotactile CCEs typically show that same-side distractors produce larger CCEs than different-side distractors, manifested as a side $\times$ congruency interaction effect (Pavani et al., 2000; Spence et al., 2004; Aspell et al., 2009). This interaction indicates that visuotactile CCEs are larger when the visual and tactile stimuli are (or are represented as being) closer to each other in space. In contrast, no such interaction effects were found in the present study, suggesting that spatial relations are not as influential for the audiotactile CCE as for the visuotactile CCE (Spence et al., 2004), which may be because spatial resolution is poorer for audition than for vision (Blauert, 1997). 


\section{Conclusions}

The present study reveals two main novel findings: (i) the first demonstration of an audiotactile crossmodal congruency effect that (ii) was only found with the current set-up when participants viewed their body. These CCEs differ from visuotactile CCEs in that they do not appear to be as sensitive to the spatial relations between the distractors and the tactile stimuli. In future work it will be important to investigate the 'self-specificity' of viewing the body on audiotactile interactions (Ehrsson, 2007; Lenggenhager et al., 2007; Aspell et al., 2009), i.e. is it necessary to view one's own body to generate this effect or is it sufficient to view another person's body or even a body-like object, such as a mannequin? Such experiments will provide further insight into personal and interpersonal multisensory body representations.

\section{Acknowledgement}

We thank H. Lissek for providing some material for the experiment.

\section{Abbreviations}

back 50 , loudspeakers at $50 \mathrm{~cm}$ behind the participants; CCE, crossmodal congruency effect; front 150, loudspeakers at $150 \mathrm{~cm}$ in front of the participants; front 50 , loudspeakers at $50 \mathrm{~cm}$ in front of the participants; HMD, head-mounted display; IE, inverse efficiency.

\section{References}

Aspell, J.E., Lenggenhager, B. \& Blanke, O. (2009) Keeping in touch with one's self: multisensory mechanisms of self-consciousness. PLoS ONE, 4 , e6488.

Austen, E., Soto-Faraco, S., Enns, J. \& Kingstone, A. (2004) Mislocalizations of touch to a fake hand. Cogn. Affect. Behav. Neurosci., 4, 170-181.

Blauert, J. 1997. Spatial Hearing, (revised edn). The MIT Press, Cambridge, MA.

Bresciani, J.-P., Ernst, M.O., Drewing, K., Bouyer, G., Maury, V. \& Kheddar, A. (2005) Feeling what you hear: auditory signals can modulate tactile tap perception. Exp. Brain Res., 162, 172-180.

Bruce, C., Desimone, R. \& Gross, C.G. (1981) Visual properties of neurons in a polysensory area in superior temporal sulcus of the macaque. J. Neurophysiol., 46, 369-384.

Caclin, A., Soto-Faraco, S., Kingstone, A. \& Spence, C. (2002) Tactile "capture" of audition. Percept. Psychophys., 64, 616-630.

Driver, J. \& Spence, C. (1998a) Attention and the crossmodal construction of space. Trends Cogn. Sci., 2, 254-262.

Driver, J. \& Spence, C. (1998b) Cross-modal links in spatial attention. Philos. Trans. R. Soc. Lond. B Biol. Sci., 353, 1319-1331.

Ehrsson, H. (2007) The Experimental Induction of Out-of-Body Experiences. Science, 317, 1048

Farne, A. \& Ladavas, E. (2002) Auditory peripersonal space in humans. J. Cogn. Neurosci., 14, 1030-1043.

Foxe, J.J., Morocz, I.A., Murray, M.M., Higgins, B.A., Javitt, D.C. \& Schroeder, C.E. (2000) Multisensory auditory-somatosensory interactions in early cortical processing revealed by high-density electrical mapping. Cogn. Brain Res., 10, 77-83.

Fu, K.-M.G., Johnston, T.A., Shah, A.S., Arnold, L., Smiley, J., Hackett, T.A., Garraghty, P.E. \& Schroeder, C.E. (2003) Auditory Cortical Neurons Respond to Somatosensory Stimulation. J. Neurosci., 23, 7510-7515.

Ghazanfar, A.A. \& Schroeder, C.E. (2006) Is neocortex essentially multisensory? Trends Cogn. Sci., 10, 278-285.

Graziano, M.S.A., Reiss, L.A.J. \& Gross, C.G. (1999) A neuronal representation of the location of nearby sounds. Nature, 397, 428-430.

Haggard, P., Christakou, A. \& Serino, A. (2007) Viewing the body modulates tactile receptive fields. Exp. Brain Res., 180, 187-193.

Igarashi, Y., Kimura, Y., Spence, C. \& Ichihara, S. (2008) The selective effect of the image of a hand on visuotactile interactions as assessed by performance on the crossmodal congruency task. Exp. Brain Res., 184, 31-38.

Jousmäki, V. \& Hari, R. (1998) Parchment-skin illusion: sound-biased touch. Curr. Biol., 8, R190-R191.
Kayser, C., Petkov, C.I., Augath, M. \& Logothetis, N.K. (2005) Integration of touch and sound in auditory cortex. Neuron, 48, 373-384.

Kennett, S., Taylor-Clarke, M. \& Haggard, P. (2001) Noninformative vision improves the spatial resolution of touch in humans. Curr. Biol., 11, 11881191.

Kitagawa, N. \& Spence, C. (2006) Audiotactile multisensory interactions in human information processing. Jpn. Psychol. Res., 48, 158-173.

Kitagawa, N., Zampini, M. \& Spence, C. (2005) Audiotactile interactions in near and far space. Exp. Brain Res., 166, 528-537.

Leinonen, L., Hyvarinen, J. \& Sovijarvi, A.R.A. (1980) Functional properties of neurons in the temporo-parietal association cortex of awake monkey. Exp. Brain Res., 39, 203-215.

Lenggenhager, B., Tadi, T., Metzinger, T. \& Blanke, O. (2007) Video Ergo Sum: Manipulating Bodily Self-Consciousness. Science, 317, 1096-1099.

Lloyd, D., Merat, N., McGlone, F. \& Spence, C. (2003) Crossmodal links between audition and touch in covert endogenous spatial attention. Percept. Psychophys., 65, 901-924.

Lütkenhöner, B., Lammertmann, C., Simões, C. \& Hari, R. (2002) Magnetoencephalographic Correlates of Audiotactile Interaction. NeuroImage, 15, 509-522.

Menning, H., Ackermann, H., Hertrich, I. \& Mathiak, K. (2005) Spatial auditory attention is modulated by tactile priming. Exp. Brain Res., 164, 4147.

Pavani, F. \& Castiello, U. (2004) Binding personal and extrapersonal space through body shadows. Nat. Neurosci., 7, 14-16.

Pavani, F., Spence, C. \& Driver, J. (2000) Visual capture of touch: out-of-thebody experiences with rubber gloves. Psychol. Sci., 11, 353-359.

Schicke, T., Bauer, F. \& Röder, B. (2009) Interactions of different body parts in peripersonal space: how vision of the foot influences tactile perception at the hand. Exp. Brain Res., 192, 703-715.

Schroeder, C.E. \& Foxe, J. (2005) Multisensory contributions to low-level, [']unisensory' processing. Curr. Opin. Neurobiol., 15, 454-458.

Schürmann, M., Caetano, G., Hlushchuk, Y., Jousmäki, V. \& Hari, R. (2006) Touch activates human auditory cortex. NeuroImage, 30, 1325-1331.

Shore, D.I., Barnes, M.E. \& Spence, C. (2006) Temporal aspects of the visuotactile congruency effect. Neurosci. Lett., 392, 96-100.

Soto-Faraco, S. \& Deco, G. (2009) Multisensory contributions to the perception of vibrotactile events. Behav. Brain Res., 196, 145-154.

Spence, C., Kingstone, A., Shore, D.I. \& Gazzaniga, M.S. (2001) Representation of visuotactile space in the split brain. Psychol. Sci., 12, 90-93.

Spence, C., Pavani, F. \& Driver, J. (2004) Spatial constraints on visual-tactile cross-modal distractor congruency effects. Cogn. Affect. Behav. Neurosci., 4, $148-169$.

Stein, B. \& Meredith, M. (1993) The Merging of the Senses. MIT Press, Cambridge, MA

Tajadura-Jiménez, A., Kitagawa, N., Väljamäe, A., Zampini, M., Murray, M.M. \& Spence, C. (2009) Auditory-somatosensory multisensory interactions are spatially modulated by stimulated body surface and acoustic spectra Neuropsychologia, 47, 195-203.

Tipper, S., Lloyd, D., Shorland, B., Dancer, C., Howard, L. \& McGlone, F. (1998) Vision influences tactile perception without proprioceptive orienting. Neuroreport, 9, 1741-1744.

Tipper, S., Phillips, N., Dancer, C., Lloyd, D., Howard, L. \& McGlone, F. (2001) Vision influences tactile perception at body sites that cannot be viewed directly. Exp. Brain Res., 139, 160-167.

Townsend, J. \& Ashby, F. (1983) Stochastic Modelling of Elementary Psychological Processes. Cambridge University Press, New York.

Wallace, M.T., Meredith, M.A. \& Stein, B.E. (1993) Converging influences from visual, auditory, and somatosensory cortices onto output neurons of the superior colliculus. J. Neurophysiol., 69, 1797-1809.

Warren, D. (1970) Intermodality interactions in spatial localization. Cogn. Psychol., 1, 114-133.

Welch, R.B. \& Warren, D.H. (1986) Intersensory interactions. In Boff, K.R., Kaufman, L. \& Thomas, J.P. (Eds), Handbook of Perception and Human Performance-Sensory Processes and Perception. Wiley, New York, pp. 1-36.

Whiteley, L., Kennett, S., Taylor-Clarke, M. \& Haggard, P. (2004) Facilitated processing of visual stimuli associated with the body. Perception, 33, $307-$ 314.

Whiteley, L., Spence, C. \& Haggard, P. (2008) Visual processing and the bodily self. Acta Psychol., 127, 129-136.

Zampini, M., Brown, T., Shore, D.I., Maravita, A., Röder, B. \& Spence, C. (2005) Audiotactile temporal order judgments. Acta Psychol., 118, 277-291.

Zampini, M., Torresan, D., Spence, C. \& Murray, M.M. (2007) Auditorysomatosensory multisensory interactions in front and rear space. Neuropsychologia, 45, 1869-1877. 